\title{
CÓMO RESPONDEN A LA CRISIS ECONÓMICA ACTUAL LAS SOCIEDADES COOPERATIVAS AGRARIAS. EL CASO DE LAS ALMAZARAS COOPERATIVAS ANDALUZAS.
}

\author{
POR \\ Carmen RUIZ JIMÉNEZ1, \\ Elia GARCÍA MARTí ${ }^{2}$ y \\ Ma Jesús HERNÁNDEZ ORTIZ ${ }^{3}$
}

\section{RESUMEN}

Ante la complejidad del entorno actual, caracterizado por la crisis económica, el modelo empresarial cooperativo se presenta como una opción con garantías de éxito para hacer frente a la situación y poder competir en el mercado. El objetivo de este trabajo es comprobar de qué forma las cooperativas agrarias andaluzas están manteniéndose en esta etapa crítica. Es decir, tratamos de identificar qué factores, qué recursos permiten que la cooperativa pueda crear determinadas capacidades y mantener su competitividad en el mercado en momentos de crisis.

Para conseguir este objetivo el trabajo ofrece una visión cualitativa del efecto de la crisis sobre las cooperativas almazareras en Andalucía, basada en la experiencia y el conocimiento de los responsables de las distintas empresas cooperativas. En el trabajo se profundiza en diferentes aspectos, fundamentalmente en las características, que por su propia naturaleza, poseen las organizaciones cooperativas y en las medidas que estás han adoptado para hacer frente a la crisis económica actual.

\footnotetext{
${ }^{1}$ Carmen Ruiz Jiménez: Departamento Organización de Empresas, Universidad de Jaén. Dirección de correo electrónico: cruiz@ujaen.es.

${ }^{2}$ Elia García Martí: Departamento Organización de Empresas, Universidad de Jaén. Dirección de correo electrónico: egarcia@ujaen.es.

${ }^{3} \mathrm{M}^{\mathrm{a}}$ Jesús Hernández Ortiz: Departamento Organización de Empresas, Universidad de Jaén. Dirección de correo electrónico: mjhernan@ujaen.es.
}

REVESCO No 113 - MONOGRÁFICO: Crisis económica actual y sus posibles repercusiones en la economía social - ISSN: 1885-8031 - www.ucm.es/info/revesco 
Palabras clave: Sociedades cooperativas agrarias, crisis económica, competitividad, teoría de recursos y capacidades, teoría relacional.

Claves Econlit: M100, Q130, L140, G300, G390.

\title{
HOW DO THE AGRARIAN COOPERATIVES ANSWER TO THE ECONOMIC CRISIS. THE CASE OF THE ANDALUSIAN COOPERATIVE OLIVE-OIL
}

\begin{abstract}
Due to the current environment, characterized by the economic crisis, the cooperative managerial model it presents itself as an option with success guarantees, to face the situation and power to compete on the market. The purpose of this paper is to verify of what form the Andalusian agrarian cooperatives are supported in this critical stage. We try to identify what factors, what resources allow the cooperative to be able to create certain capacities and maintain its competitiveness on the market in crisis.

To obtain this purpose, the paper offers a qualitative vision of the effect of the crisis on the olive oil cooperatives in Andalusia based on the experience and the knowledge of the persons in charge of the different cooperatives. In the paper, we analyses in depth different aspects, fundamentally the characteristics, which for their own nature the cooperative has and the measurements that they have adopted to face to the current economic crisis.
\end{abstract}

Keywords: Agrarian cooperatives, economic crisis, competitiveness, resources and capabilities theory, relational theory.

\section{INTRODUCCIÓN}

La Asamblea General de las Naciones Unidas declaró el pasado año 2012 como el año Internacional de las Cooperativas pretendiendo llamar la atención sobre las inestimables contribuciones de las empresas cooperativas a la reducción de la pobreza, la generación de empleo y la integración social. En palabras de Ban Ki-moon, secretario general de Naciones Unidas: "Con su distintivo énfasis en los valores, las cooperativas han demostrado ser un modelo empresarial versátil y viable, que puede prosperar incluso en épocas difíciles. Su éxito ha contribuido a impedir que muchas familias y comunidades caigan en la pobreza”. A raíz de

REVESCO $\mathrm{N}^{\circ} 113$ - MONOGRÁFICO: Crisis económica actual y sus posibles repercusiones en la economía social - ISSN: 1885-8031 - www.ucm.es/info/revesco 
esta celebración son numerosos los artículos que tratan de corroborar este hecho y mostrar la situación del cooperativismo a nivel internacional y nacional.

Se constata, por los datos que muestran diferentes instituciones, que las cooperativas tienen una probada trayectoria en la creación y mantenimiento del empleo, tratándose de un modelo empresarial que durante la profunda crisis económica mundial que sufrimos continúa generando empleo. Se subraya que el cooperativismo está cubriendo alguna de las funciones sociales que el estado de bienestar está dejando de asumir por la crisis: atención sanitaria, cuidado de dependientes y discapacitados, servicios financieros, enseñanza, energía renovable, actividades culturales, intermediación laboral, agricultura... ${ }^{4}$.

También son numerosos los trabajos a escala internacional que inciden en el carácter contracíclico en la dinámica del empleo. El informe CECOP-CICOPA (2011) que analiza el impacto de la crisis actual sobre las cooperativas en distintos países de Europa, evidencia una mejor situación en términos de rendimiento económico, tasa de supervivencia y empleo en las cooperativas, que en las empresas convencionales (Calderón y Calderón, 2012). En relación a etapas críticas anteriores, Guerreri et al. (1992) afirman que, en Italia, donde la ocupación total apenas creció un $1 \%$ al año, en las cooperativas lo hizo a una tasa del 5,7\%. Cornforth y Thomas (1995) señalan que la economía francesa apenas pudo mantener el empleo, sin embargo, los puestos de trabajo en cooperativas se multiplicaron por más de 1,4. En el Reino Unido y Holanda el empleo en las cooperativas experimentó un crecimiento más que espectacular al multiplicarse casi por ocho entre 1970 y 1985 (Calderón y Calderón, 2012).

En concreto, las cooperativas agroalimentarias crean empleo y facturan más a pesar de la crisis actual, así desde 2006 a 2011 han incrementado un 6,7\% la creación de empleo y un $13 \%$ el volumen de facturación ${ }^{5}$, permaneciendo estable el número de socios. La comunidad que cuenta con un mayor número de cooperativas en este sector es Andalucía con 736, seguida de Castilla-La Mancha, Castilla-León y Comunidad Valenciana.

En el caso concreto del sector del aceite de oliva, actualmente España es la primera potencia mundial, ya que produce de media entre un 40 y un 60 por ciento del aceite, dependiendo de la campaña, y consume el 20 por ciento del total. El olivo es un importante pilar de la sociedad en las diferentes regiones en las que se cultiva. Genera anualmente unos

\footnotetext{
${ }^{4}$ FAECTA COOP. 13 de marzo de 2013.

${ }^{5}$ En ABC economía. 24 de mayo de 2013.
}

REVESCO No 113 - MONOGRÁFICO: Crisis económica actual y sus posibles repercusiones en la economía social - ISSN: 1885-8031 - www.ucm.es/info/revesco 
46 millones de días de trabajo, siendo por tanto una importante fuente de empleo (el 1,5 por ciento de la población activa nacional), sobre todo en regiones con altas tasas de desempleo, donde el olivo es realmente un monocultivo. A estos efectos positivos sobre el empleo, hay que añadir los producidos por la industria, la comercialización y el embalaje. Además de la importancia socio-económica de los productos oleícolas, el esfuerzo realizado en investigación y desarrollo en las últimas décadas ha situado a España en la vanguardia de la investigación y el desarrollo tecnológico en esta área, provocando que no sea solo el primer productor en cuanto a volumen sino también en cuanto a calidad y diversidad del producto (Cardenas y Vilar, 2012).

Los principales datos de mercado del sector del aceite de oliva se resumen en el siguiente balance de las 5 últimas campañas, tabla 1:

Tabla 1

\begin{tabular}{llllll}
\hline 'Campañas & $\mathbf{2 0 0 6 / 2 0 0 7}$ & $\mathbf{2 0 0 7 / 2 0 0 8}$ & $\mathbf{2 0 0 8 / 2 0 0 9}$ & $\mathbf{2 0 0 9 / 2 0 1 0}$ & $\mathbf{2 0 1 0 / 2 0 1 1}$ \\
\hline -stock inicial & 288.400 & 331.800 & 425.700 & 285.700 & 420.700 \\
producción & 1.111 .400 & 1.236 .100 & 1.030 .000 & 1.401 .500 & 1.390 .500 \\
importaciones & 83.400 & 61.400 & 38.900 & 47.000 & 43.400 \\
\hline TOTAL DISPONIBLE & 1.483 .200 & 1.629 .300 & 1.494 .600 & 1.734 .200 & 1.854 .600 \\
Consumo y perdidas & 560.000 & 537.500 & 533.600 & 533.400 & 553.400 \\
exportaciones & 591.400 & 666.100 & 675.300 & 780.100 & 827.700 \\
TOTAL SALIDAS & 1.151 .400 & 1.203 .600 & 1.208 .900 & 1.313 .500 & 1.381 .100 \\
STOK FINAL & 331.800 & 425.700 & 285.700 & 420.700 & 473.500 \\
Precio medio (Pool & $2.484,39$ & $2.444,13$ & $1.936,84$ & $1.886,10$ & $1.803,16$ \\
\hline
\end{tabular}

Fuente: Balance 2001, sector aceite de oliva, cooperativas agro-alimentarias.

Hay que destacar que las exportaciones suponen ya el $60 \%$ de las salidas de aceite y van en aumento. España se ha situado por delante de Italia como principal país exportador mundial y se va consolidando en la mayoría de los países, como el número uno en cuota de mercado. En consecuencia, la primera conclusión a la que podemos llegar es que es un sector productivo que contribuye a generar riqueza y empleo de forma sostenible. Estas circunstancias, sobre todo en tiempos de crisis, deberían ser suficientes para que se reconozca su importancia y se le conceda el carácter estratégico que se merece.

En contrapartida, los precios del aceite de oliva en origen son los más bajos de las 10 últimas campañas y se sitúan muy por debajo del coste de producción. Y además, a modo de ver de gran parte de los implicados, el trance por el que pasa el sector en su conjunto, no es pasajero, sino estructural al afectar a las mismas entrañas de la totalidad del sector agroalimentario.

REVESCO No 113 - MONOGRÁFICO: Crisis económica actual y sus posibles repercusiones en la economía social - ISSN: 1885-8031 - www.ucm.es/info/revesco 
En este sentido, podemos apuntar que este sector tiene una estructura muy atomizada que se debe corregir urgentemente a fin de ganar competitividad y eficiencia. Según el Balance 2011 de Cooperativas Agroalimentarias, en la campaña 2010/11 el número de almazaras cooperativas con actividad fue de 947 sobre un total de 1.744 almazaras y la producción de almazaras cooperativas fue de 945.000 toneladas de un total de 1.390.000 toneladas. 730 almazaras cooperativas desarrollan actividad de envasado aunque la mayor parte es para abastecer a sus socios y mercados locales. Se han consolidado 15 grupos de comercialización, de diferente dimensión, que agrupan en torno a 250 almazaras cooperativas.

Las cooperativas se han apuntado a la corriente exportadora, incrementando notablemente sus exportaciones, donde se consiguen precios más elevados que en el mercado interior. Por el contrario, en el mercado interior la presión de la marca de la distribución con precios incluso por debajo de coste, ha ido desplazando del mercado a las marcas de fabricante lo que ha supuesto que las cooperativas hayan mantenido su cuota de mercado, o incluso haya disminuido a favor de la marca de distribución. Asimismo, dado que en las últimas campañas, hay una mayor diferencia de precios entre las distintas categorías de aceite de oliva, las cooperativas que han apostado por la calidad consiguen mejores liquidaciones a sus socios.

La crisis económica sigue marcando la pauta de los operadores con operaciones a muy corto plazo y pequeños volúmenes, en las que la gran competencia entre grupos de distribución, con el aceite de oliva como producto reclamo, está impidiendo una mejora de los precios en origen. El sector productor es consciente de que necesita organizarse para concentrar la oferta y poder negociar en igualdad de condiciones con los envasadores y la gran distribución. Los procesos de concentración cooperativos de los últimos años (Dcoop ${ }^{6}$, Jaencoop, Interoleo Picual, etc.) muestran esta tendencia.

Los resultados anteriores se explican ${ }^{7}$ considerando que el modelo empresarial de las cooperativas tiene elementos clave para competir en entornos complejos. El enfoque basado en los recursos señala que determinados resultados se pueden alcanzar al disponer de los recursos adecuados y hacer un buen uso de los mismos. Esto nos remite a la necesidad de considerar cual es el papel de la dotación de recursos en la competitividad de estas empresas.

\footnotetext{
${ }^{6}$ Véase en este sentido: Hernandez, Marquez Y Pedrosa (2012)

${ }^{7}$ Sabin Galán et al. (2012); El País, Cataluña, 29/09/12; ABC. es,13/3/2013.
}

REVESCO No 113 - MONOGRÁFICO: Crisis económica actual y sus posibles repercusiones en la economía social - ISSN: 1885-8031 - www.ucm.es/info/revesco 
Más aún cuando los recursos y capacidades estratégicos de la empresa, pueden jugar un papel ampliamente relevante como moderadores de la crítica situación económica.

A este respecto las sociedades cooperativas pueden plantear diferencias significativas entre su dotación y utilización de recursos y una empresa convencional. Estas diferencias, que en algunos casos pueden suponer limitaciones, en otros les proporcionará ventajas. Del mismo modo, la teoría relacional nos puede servir para ampliar nuestro análisis. El concepto de capital social es básico para el análisis de la competitividad de las empresas. En particular, en las cooperativas, ha sido utilizado para evaluar la calidad de las relaciones, incluyendo la confianza, las normas relacionales y las redes sociales como aspectos que pueden ayudar a mejorar la efectividad de dichas relaciones (Spear, R., 2000).

Teniendo en cuenta lo anterior, en este trabajo se pretende avanzar en el conocimiento de los factores en los que se basa la competitividad de las sociedades cooperativas en épocas de crisis, profundizando en sus características específicas ${ }^{8}$. Concretamente, nuestro análisis se centra en indagar en relación al papel que pueden jugar algunos elementos, desde los que la literatura se ha aproximado al concepto de competitividad, como son el entorno o las características internas de las empresas. Esto puede servir para comprender, desde una perspectiva amplia, el contexto asociado a la existencia y el desarrollo de las cooperativas.

Siguiendo la corriente de dirección estratégica sobre gestión del conocimiento aplicado a nivel macroorganizativo, intentamos movilizar el conocimiento obtenido en las organizaciones para generar nuevo conocimiento que las sociedades cooperativas necesitan para estar (o seguir) en primera línea competitiva.

La insuficiente investigación en este ámbito y la necesidad de analizar el modelo empresarial de las cooperativas en su contexto, en su ambiente natural, nos ha llevado a desarrollar un estudio de carácter cualitativo a partir del análisis de un caso, el de las almazaras cooperativas andaluzas, para adentrarnos en su funcionamiento interno y analizar sus pautas de gestión.

Llegados a este punto, resulta preceptivo plantear las siguientes cuestiones:

\footnotetext{
${ }^{8}$ A diferencia del resto de las empresas capitalistas, en la cultura de las cooperativas inciden de forma muy significativa una serie de principios y valores que orientan y marcan la forma de comportarse de estas organizaciones.
}

REVESCO No 113 - MONOGRÁFICO: Crisis económica actual y sus posibles repercusiones en la economía social - ISSN: 1885-8031 - www.ucm.es/info/revesco 
¿Cómo y a qué tipo de recursos acceden los agricultores a través de las cooperativas?

¿Cómo generan las capacidades empresariales que les permiten mantenerse en una situación tan adversa?

¿Qué atributos singulares reúne el capital social de una cooperativa agroalimentaria?

La estructura del presente trabajo es la siguiente: el próximo apartado desarrolla la fundamentación teórica, donde se recogen los objetivos de investigación. A continuación, se detalla la metodología empleada, se presentan y discuten los resultados del caso de estudio, finalizando el trabajo con las conclusiones en las que se recogen las distintas respuestas de las sociedades cooperativas agrarias ilustradas a partir del caso de las almazaras cooperativas andaluzas.

\section{FUNDAMENTACIÓN TEÓRICA}

Las sociedades cooperativas logran ubicar a las personas en el centro del análisis de la actividad económica, desplazando la utilización del capital como objeto básico de estudio. El movimiento cooperativo logra establecer un modelo organizacional sustentado sobre unos principios, en los cuales prima la humanización de las actividades económicas (GarcíaGutiérréz, 2002; Lejarriega, 2002). Los principios cooperativos tienen el potencial de guiar estrategias y prácticas para poder ser convertidos en ventajas cooperativas (Novkovic, 2008).

Es un hecho que las cooperativas, y el movimiento cooperativo en sí, poseen unos valores sociales, y que dichos valores son distintos a los de otras formas de organización empresarial. Son estas diferencias las que plantean que organizaciones diferentes a las empresas convencionales deben ser analizadas en base a criterios más amplios o diferentes, con el fin de entregar conclusiones que se ajusten a su naturaleza y funcionamiento (Marcuello y Nachar, 2013).

La economía de organizaciones ha estudiado el fenómeno asociado a la conformación y desarrollo de las sociedades cooperativas desde sus diversas perspectivas teóricas, centradas principalmente en las teorías de derechos de propiedad y de costes de agencia, llegando a un consenso en lo que respecta a las desventajas con las que cuenta este tipo de organizaciones a la hora de participar de la economía de mercado. No obstante, muchas de las desventajas institucionales del modelo cooperativo organizacional responden más bien al análisis teórico

REVESCO No 113 - MONOGRÁFICO: Crisis económica actual y sus posibles repercusiones en la economía social - ISSN: 1885-8031 - www.ucm.es/info/revesco 
(Bartlett, et al., 1992). En este sentido, la inclusión de un enfoque más amplio permite poder ver la forma de solucionar los inconvenientes identificados por la literatura neoclásica, además de brindar la oportunidad de añadir ventajas tales como la creación de capital social y la reducción de externalidades negativas, siendo necesario para esto un marco en el cual se incluyan dentro del análisis los roles característicos, posibles de cumplir, al ser una organización que cuenta con una serie de valores y principios (Novkovic, 2008).

De este modo, la literatura de economía de las organizaciones debe ampliar su perspectiva de análisis, considerando la capacidad de este tipo de organizaciones de generar un impacto positivo en la sociedad, bajo la forma de aminorar externalidades negativas generadas por los ciclos económicos o fallos de mercado (Jones y Kalmi, 2009), y bajo la premisa de creación de capital social, el cual contribuye positivamente en el desarrollo de una sociedad más justa y solidaria (Novkovic, 2008, Marcuello y Saz, 2008).

La Teoría de Recursos y Capacidades (TRC) plantea la existencia de una relación directa entre los recursos y capacidades de una organización y su posición competitiva. Su premisa fundamental es la existencia de heterogeneidad entre las empresas en cuanto a los recursos que controlan, siendo dicha heterogeneidad la que explica los diferentes resultados obtenidos por cada una de ellas (Barney, 1991). El problema de acceso a los recursos ha sido ampliamente tratado por diferentes autores. Teece (1987) propone un modelo en el que desde la perspectiva de la TRC se analiza la conveniencia de las diferentes formas de acceder a los mismos: desarrollo interno, adquisición en el mercado y realización de acuerdos de colaboración, escogiendo una u otra forma en función de la naturaleza de los recursos complementarios, de las oportunidades que presenten y de los costes de transacción que conllevan.

Los acuerdos de colaboración se hacen necesarios para la incorporación de recursos y capacidades, sobre todo en entornos complejos y globalizados, por requerir de menor tiempo que el desarrollo interno y tener mayor flexibilidad que la adquisición. Entre los acuerdos de colaboración en el ámbito agroalimentario figuran las cooperativas agroalimentarias. A través de ellas los agricultores pueden acceder a una serie de recursos con los que hacer frente a diferentes condicionantes (técnicos, humanos, económicos y políticos) de la actividad agraria, y responder a las cada vez mayores exigencias de las cadenas de distribución y de los consumidores finales (Arcas et al., 2011).

REVESCO No 113 - MONOGRÁFICO: Crisis económica actual y sus posibles repercusiones en la economía social - ISSN: 1885-8031 - www.ucm.es/info/revesco 
Así, nos preguntamos si la cooperativa puede ser una forma eficiente de resolver los problemas de agencia y de costes de transacción, al poder desarrollar recursos y capacidades que le hagan participar en el mercado de forma eficiente, compitiendo bajo un esquema en el que se colabora internamente y que mediante la confianza entre las personas se pueden asumir menores costes, tanto económicos como sociales, y generar externalidades positivas en la situación económica tan precaria en la que nos encontramos.

Los objetivos de nuestra investigación son: a) analizar cómo y a qué tipo de recursos acceden los agricultores a través de las cooperativas; b) indagar la forma en que los utilizan y los combinan generando capacidades empresariales que les permiten mantenerse en una situación tan adversa y les ayudan a satisfacer sus expectativas relativas a la producción y comercialización de sus productos para conseguir sus objetivos. Esto puede ayudar a generar conocimiento para poder ser aplicado en otro tipo de empresas de participación, ya que encontramos pocas evidencias del comportamiento real de estas organizaciones en momentos de crisis.

Existen indicios suficientes que apuntan que detrás de las prácticas de gestión de las empresas de participación y especialmente de las cooperativas, se encuentran la cultura y la filosofía cooperativa. No obstante, encontramos muy pocas evidencias sobre cuáles son los recursos y capacidades más significativos que les permiten mantenerse en el mercado en situaciones adversas.

Del mismo modo, la teoría relacional nos puede servir para ampliar nuestro análisis. Así, esta teoría parte de la fundamentación teórica en la que la relación entre los partícipes de una empresa está incluida en un contexto relacional o social. El concepto de capital social es básico para el análisis de las empresas. En particular, para las cooperativas ha sido utilizado para evaluar la calidad de las relaciones, incluyendo la confianza, las normas relacionales y las redes sociales como aspectos que pueden ayudar a mejorar la efectividad de dichas relaciones (Spear, R., 2000).

El capital social puede ser definido como las normas, valores, actitudes y creencias que presiden la relación socio-cooperativa, destacando la confianza que la empresa cooperativa inspira a los socios y la justicia que estos perciben en la relación con la cooperativa (Arcas et al., 2011). Rodríguez-Mondoño (2012) indica que el capital social incluye un conjunto de normas y organizaciones sociales que facilitan la cooperación de los REVESCO No 113 - MONOGRÁFICO: Crisis económica actual y sus posibles repercusiones en la economía social - ISSN: 1885-8031 - www.ucm.es/info/revesco 
agentes, en la persecución de un beneficio común y tienen impacto en la eficacia y eficiencia de una comunidad.

Entre otras definiciones de capital social encontramos la de Nahapiet y Ghoshal (1998), como la "suma de recursos actuales y potenciales derivados de la red de relaciones de la unidad social". O la de IADE (2003) como "el valor que representan para la empresa las relaciones que mantiene con los agentes sociales que actúan en su ámbito, en términos del nivel de integración, compromiso, cooperación, cohesión, conexión y responsabilidad social que establece con la sociedad". En definitiva, el capital social implica el establecimiento de una red de relaciones, proporciona una estructura para la circulación de información y representa un bien colectivo que reduce los costes de transacción (Rodríguez, 2004).

El capital social en la empresa cooperativa, está recogido en los principios cooperativos compartidos por todos sus miembros, que constituyen una herramienta de gestión y gobierno que diferencia a este tipo de empresa del resto y a su vez, le otorga una ventaja competitiva en momentos como el actual de crisis económica. Esta realidad la vemos reflejada en la estructura de propiedad de estas empresas, que facilita el reparto de sacrificios en aras de la continuidad de la empresa. En la gestión democrática que propicia la toma de decisiones dialogadas lo que da mayor flexibilidad a la empresa. Y en el fomento de la intercooperación y el trabajo en red, como medio de supervivencia para adaptarse a las nuevas circunstancias.

Dicho capital social se puede considerar como un intangible de elevador valor. Actualmente, los activos intangibles basados en el conocimiento y en los recursos humanos, representan una de las mayores fuentes de creación de valor en las organizaciones siendo la base del éxito competitivo de las empresas. De este modo, la identificación y evaluación de dicho intangible es importante desde el punto de vista de la gestión empresarial. Como afirman Spender y Grant (1996: 8) las variables más relevantes desde el punto de vista teórico para la gestión estratégica son precisamente aquellas menos identificables y menos cuantificables.

Dentro del conjunto de conocimientos organizativos, que se incluyen en el capital social, nos encontramos a nivel de análisis grupal con el capital relacional que se apoya en la confianza y la justicia presentes en la organización, como ya hemos puesto de manifiesto con anterioridad. La base del capital relacional es el conocimiento acumulado por las partes en los REVESCO No 113 - MONOGRÁFICO: Crisis económica actual y sus posibles repercusiones en la economía social - ISSN: 1885-8031 - www.ucm.es/info/revesco 
intercambios con terceros. Se puede medir en función de la longevidad, por lo que este recurso adquiere mayor valor cuando aumenta la duración de las relaciones con clientes, proveedores, accionistas, etc. Y, a causa de su naturaleza, el capital relacional es el conocimiento que más dificultades de codificación presenta (Bontis, 2001).

De este modo, el capital relacional incluye no solo los intercambios internos de conocimiento entre los empleados y la transferencia de conocimiento entre estos empleados y las estructuras y sistemas organizativos, sino también los intercambios de conocimiento entre los empleados y los proveedores, los clientes, los socios de alianzas, etc. (Bontis, 2001; Stewart, 1997; Youndt et al., 2004).

El capital relacional se define como el valor de los conocimientos que se incorporan a las personas y a la organización derivados de las relaciones -más o menos permanentes- que mantienen con los agentes del mercado y con la sociedad en general (IADE, 2003). Por lo que en la definición de capital relacional se incluye todo el valor generado por las relaciones que la organización mantiene con sus grupos de referencia, tanto con sus grupos de interés internos como externos (Bontis, 1996).

En la empresa cooperativa podemos apreciar la importancia del capital relacional en diversos aspectos: en la flexibilidad de la empresa para adaptarse a las condiciones del mercado; en el fomento de la intercooperación y el trabajo en red, como medio de supervivencia ante las nuevas circunstancias; y en la creación de empresas como entidades cualificantes de los miembros que las componen.

Todo lo anterior se puede apoyar en la confianza en la empresa, que puede ser definida como la creencia o percepción de los socios de que las intenciones de la cooperativa a la que pertenecen, le llevará a adoptar comportamientos que le permitirán conseguir los resultados previstos (Arcas et. al, 2011). Para Aulakh et al. (1996) la literatura identifica tres grandes consecuencias de la confianza en las relaciones interorganizacionales. En primer lugar, disminuye el comportamiento oportunista y fomenta la cooperación, ya que las empresas renuncian a las ganancias individuales a corto plazo en favor de los beneficios a largo plazo de mantener la relación. En segundo lugar, tiene implicaciones positivas en los resultados de las empresas en el mercado y en la eficiencia. Por último, es considerada como un sustituto del gobierno basado en la jerarquía, cuando el control basado en la propiedad resulta inviable desde el punto de vista económico o estratégico.

REVESCO No 113 - MONOGRÁFICO: Crisis económica actual y sus posibles repercusiones en la economía social - ISSN: 1885-8031 - www.ucm.es/info/revesco 
Existen trabajos que confirman la influencia positiva de la confianza en la satisfacción en las relaciones interorganizacionales (Anderson y Narus, 1990; Andaleeb, 1996; Iglesias et al., 1998) y, en particular en el ámbito de las cooperativas agroalimentarias (Arcas, 2003). Del mismo modo, en el ámbito de las cooperativas agroalimentarias también están apareciendo trabajos que se centran en la importancia de la justicia en la relación (Borgen, 1999; Arcas et al., 2000; Hernández y Arcas, 2003). Ponen de manifiesto que el trato justo e igualitario a los socios es uno de los valores distintivos de las empresas cooperativas, manifestándose en los principios de gestión democrática y participación económica de los socios. La justicia puede ser definida como "la percepción o sentimiento del socio acerca de la equidad de la relación que mantiene con la cooperativa y del trato que recibe de ésta”.

También se puede considerar que la productividad de una empresa depende de su capacidad para insertarse en su entorno y relacionarse de forma estratégica. Para ello las redes inter-organizacionales e intra-organizacionales basadas en la confianza y la cooperación, facilitan los procesos de creación, transferencia, aprendizaje o gestión de nuevos conocimientos en las empresas (Rodríguez-Mondoño, 2011). La importancia de las relaciones de la empresa con las partes externas contribuye a que ésta prolongue su horizonte temporal con relaciones que no se basan en transacciones puntuales, sino en intercambios de bienes e información a largo plazo (Williamson, 1975).

Así, el tercer objetivo de nuestro trabajo será analizar cómo las organizaciones cooperativas dedican parte de sus recursos a incrementar su capital social, dado que el conocimiento se encuentra en redes y grupos, siendo el capital social imprescindible para capturar el conocimiento que conduce a la innovación y a generar una ventaja competitiva para la organización. Cómo establecen "estrategias relacionales", ${ }^{9}$, buscando relaciones de privilegio con diversos agentes del entorno más allá de las relaciones normales establecidas en un contexto de mercado, con la finalidad de proteger a la empresa de la competencia, buscando "zonas de seguridad" 10 , que eliminen la incertidumbre del entorno y aseguren la supervivencia de la organización.

\footnotetext{
${ }^{9}$ Véase el significado en STRATEGOR, 1995.

${ }^{10}$ Definido el término en GUERRAS, 1998.
}

REVESCO No 113 - MONOGRÁFICO: Crisis económica actual y sus posibles repercusiones en la economía social - ISSN: 1885-8031 - www.ucm.es/info/revesco 


\section{METODOLOGIA}

Dado que la teoría no es suficiente para entender la complejidad de los comportamientos y relaciones que subyacen en las organizaciones cooperativas, es necesario estudiar dichas relaciones en el contexto en que se desarrollan y alcanzan su máximo grado de desempeño.

De este modo y para poder dar respuesta a los interrogantes planteados utilizamos el estudio de un caso de tipo ilustrativo siguiendo la tipología de casos establecida por Ryan et al. (1992). Según estos autores, los casos ilustrativos intentan elucidar las prácticas innovadoras que impregnan el desarrollo organizativo de las empresas más competitivas. La investigación empírica que se realiza en este tipo de casos centra su atención de manera casi exclusiva en la descripción del proceso de implantación de las mejores prácticas en el seno de las organizaciones.

La elección de esta metodología se fundamenta en la posibilidad de analizar el fenómeno objeto de estudio en su contexto real, utilizando múltiples fuentes de evidencia, cuantitativas y/o cualitativas simultáneamente (Villarreal y Landeta, 2007; Yin (1989); Eisenhardt (1989). En este sentido, Chetty (1996) indica que el método de estudio de caso es una metodología rigurosa, porque posibilita estudiar los fenómenos desde múltiples perspectivas y no desde la influencia de una sola variable y permite explorar de forma más profunda y obtener un conocimiento más amplio sobre cada fenómeno.

El propósito de este caso de estudio ilustrativo es determinar cuáles son las razones por las cuales, la almazara cooperativa andaluza mantiene su posición competitiva en el entorno tan incierto en el que se encuentra. Principalmente el caso de estudio ha sido seleccionado para poder responder a las necesidades no cubiertas en la revisión de la literatura. A través de este caso ilustrativo tratamos de conocer cuál es la razón o razones que están llevando a que determinadas organizaciones cooperativas mantengan su situación o, incluso, mejoren sus resultados cuando la empresa tradicional capitalista tiene pérdidas, reduce el empleo e, incluso, abandona el mercado. Qué recursos, qué capacidades, qué tipo de relaciones se generan en el seno de esta organización que difieren de otras.

Como hemos indicado anteriormente hemos elegido el caso de la almazara cooperativa andaluza. El aceite de oliva es, en el ámbito agroalimentario español y andaluz, un valor de

REVESCO No 113 - MONOGRÁFICO: Crisis económica actual y sus posibles repercusiones en la economía social - ISSN: 1885-8031 - www.ucm.es/info/revesco 
referencia. Jaén es la mayor región productora de aceite de oliva de España y del mundo, produciendo por sí sola más aceite que el segundo país productor mundial, Italia. La provincia de Jaén con 550.000 hectáreas de olivar y más de 66 millones de olivos genera el 20\% de la producción mundial de Aceite de Oliva.

Para la realización del estudio, en primer lugar contactamos con la Federación Andaluza de Empresas Cooperativas Agrarias (FAECA), en su sede de Jaén. Del total de cooperativas dedicadas a la extracción de aceite de oliva en la provincia (180), el 87\% están asociadas a FAECA. Desde esta organización se nos ha facilitado el contacto con determinadas cooperativas almazareras que se encuentran bajo los parámetros de estudio que planteamos.

Las cooperativas fueron seleccionadas tras la entrevista con el Gerente de FAECA, y con su asesoramiento, basándonos en su experiencia, buscando aquellas cooperativas singulares por sus buenas prácticas en gestión y en la implicación con sus socios para paliar los efectos negativos de la crisis. No se buscaba las de mayor dimensión, si no la implicación con sus socios. La técnica empleada para la recogida de información ha sido la entrevista en profundidad, pues es la más adecuada para obtener información acerca de cómo los sujetos actúan y reconstruyen el sistema de representaciones sociales en sus prácticas habituales. Los sujetos entrevistados han sido miembros de los consejos rectores (presidentes o directivos) de estas organizaciones puesto que, desde nuestro punto de vista, son quienes tienen una percepción más completa de la gestión de la cooperativa, pueden facilitar la información más relevante, son más accesibles físicamente y están dispuestos a informar.

REVESCO No 113 - MONOGRÁFICO: Crisis económica actual y sus posibles repercusiones en la economía social - ISSN: 1885-8031 - www.ucm.es/info/revesco 
Tabla 2: Unidad de análisis

\begin{tabular}{|l|c|c|c|}
\hline & $\begin{array}{c}\text { Vinculación con la } \\
\text { sociedad cooperativa }\end{array}$ & Dugar & \\
\hline Entrevistado 1 & Gerente & Sede de FAECA & 78,06 minutos \\
\hline Entrevistado 2 & Gerente & Sede Cooperativa & 49,30 minutos \\
\hline Entrevistado 3 & Presidente & Sede Cooperativa & 42,19 minutos \\
\hline Entrevistado 4 & Presidente & Sede Cooperativa & 23,56 minutos \\
\hline Entrevistado 5 & Presidente & Sede ASAJA & 42,00 minutos \\
\hline Entrevistado 6 & Gerente & Sede cooperativa & 20,17 minutos \\
\hline Entrevistado 7 & $\begin{array}{c}\text { Miembro del equipo } \\
\text { directivo }\end{array}$ & Sede cooperativa & 50,24 minutos \\
\hline Entrevistado 8 & Gerente & Sede cooperativa & 42,36 minutos \\
\hline
\end{tabular}

Fuente: Elaboración propia.

A modo de resumen en la siguiente tabla recogemos la ficha técnica del estudio de casos de la almazara cooperativa jiennense.

Tabla 3. Ficha técnica del estudio de caso de la almazara cooperativa jiennense

\begin{tabular}{|c|c|}
\hline $\begin{array}{l}\text { Metodología } \\
\text { investigación }\end{array}$ & $\begin{array}{l}\text { Estudio de caso único de carácter holístico (Unidad de } \\
\text { análisis simple). } \\
\text { Estudio exploratorio, ilustrativo y potencialmente } \\
\text { explicativo. }\end{array}$ \\
\hline Unidad de análisis & La almazara cooperativa \\
\hline Ámbito geográfico & Jaén y Málaga (Andalucía) \\
\hline Universo & $\begin{array}{l}\text { Sociedades cooperativas agrarias de primer grado } \\
\text { dedicadas a la elaboración de aceite de oliva }\end{array}$ \\
\hline $\begin{array}{l}\text { Método de recogida de la } \\
\text { información }\end{array}$ & $\begin{array}{ll}\text { - } & \text { Entrevistas en profundidad, abiertas } \\
\text { semiestructuradas } & \\
\text { - } & \text { Observación directa }\end{array}$ \\
\hline Informantes clave & Miembros del consejo rector y directivos \\
\hline $\begin{array}{l}\text { Métodos de análisis de la } \\
\text { evidencia }\end{array}$ & $\begin{array}{l}\text { Fundamentalmente de tipo cualitativo: } \\
\text { - Identificación } \quad \text { y clasificación }\end{array}$ \\
\hline
\end{tabular}

REVESCO $\mathrm{N}^{\mathrm{o}} 113$ - MONOGRÁFICO: Crisis económica actual y sus posibles repercusiones en la economía social - ISSN: 1885-8031 - www.ucm.es/info/revesco 


\begin{tabular}{|l|l|}
\hline & \multicolumn{1}{|c|}{ dimensiones clave } \\
& - Búsqueda de factores explicativos clave \\
& $-\quad$ Búsqueda de patrón de comportamiento común \\
& $-\quad$ Creación de explicación teórica \\
& $-\quad$ Identificación de relaciones causales (si es posible) \\
& $-\quad$ Análisis de decisiones críticas \\
\hline Enfoque científico & $\begin{array}{l}\text { Procesos deductivos en la medida que se parte de } \\
\text { proposiciones teóricas de la revisión de teorías }\end{array}$ \\
\hline Evaluación del rigor y & Validez (constructiva, interna y externa), fiabilidad, \\
calidad metodológica & consistencia (contextual y teórico interpretativa) \\
\hline Fecha de realización & Abril-Septiembre 2013 \\
\hline
\end{tabular}

Fuente: Adaptado de Villarreal y Landeta (2007).

El análisis de la información obtenida en los discursos de los sujetos entrevistados nos ofrece los siguientes resultados, en relación a nuestros objetivos de investigación.

\section{RESULTADOS DE INVESTIGACIÓN}

En este apartado recogemos los resultados de nuestro estudio empírico. Hemos tratado de concretar cómo ha afectado la crisis económica a las organizaciones cooperativas almazareras y analizar los factores diferenciales que explican la mayor capacidad de supervivencia este tipo de empresas. La información refleja expresamente las opiniones recogidas en las entrevistas realizadas a los responsables de las distintas federaciones y cooperativas indicadas anteriormente.

\subsection{Influencia de la crisis en la almazara cooperativa}

La recesión política, social y económica que estamos sufriendo desde mediados del año 2007 ha afectado sin excepción, aunque de manera distinta, a casi todos los agentes económicos tanto a nivel nacional como internacional. El sector cooperativo agrario, que padece una crisis estructural desde hace años (crítica situación debida a: las inclemencias ambientales, a las bajadas continuas de precios en origen y a la merma en las ayudas comunitarias, entre otros factores), está soportando esta situación de crisis adoptando distintas medidas.

REVESCO No 113 - MONOGRÁFICO: Crisis económica actual y sus posibles repercusiones en la economía social - ISSN: 1885-8031 - www.ucm.es/info/revesco 
Sin embargo, no todas las cooperativas agrarias están padeciendo de la misma forma. Mientras que en algunos sectores han desaparecido sociedades cooperativas debido principalmente a que su reducida dimensión no les permite adoptar medidas con las que puedan mejorar sus niveles de rentabilidad, en el sector oleícola se mantiene el peso de la estructura del sector cooperativo, no reducen el empleo e incluso aumentan su facturación. En palabras del gerente de FAECA:

"En el aceite se están haciendo muchas fusiones. Entonces la cooperativa no desaparece. Si en un pueblo había dos y se juntan, ahora hay una solo pero con la misma capacidad que las otras dos y con menores costes para todos los socios...”.

Realmente el efecto directo de la actual situación sobre las cooperativas agrarias ha sido la reducción de ingresos que está padeciendo el socio debido a la bajada de precios y la reducción de las ayudas comunitarias. En el sector oleícola, ambos factores están llevando a muchas explotaciones al borde de la rentabilidad. Esta circunstancia está obligando a una paulatina reconversión de las plantaciones de olivar tradicional hacia el cultivo intensivo (cuyos costes de recolección son mucho menores), en aquellos casos en los que es viable para el agricultor e incluso, como nos indica uno de los sujetos, de agricultores que proceden de otros cultivos.

"Aquí llevamos recibiendo a socios en los últimos tres años que vienen de la cereza. Han quitado los cerezos y lo están llenado todo de olivar intensivo que les cuesta tres pesetas la recolección".

\subsection{Factores básicos indicadores de competitividad en épocas de crisis}

En este apartado recogemos algunos de los factores que a raíz del análisis de los discursos mantenidos con los entrevistados, podemos considerar que responden a nuestra principal cuestión: ¿Por qué las cooperativas almazareras resisten mejor la crisis? ¿Qué indicadores de competitividad hacen especialmente relevante a este tipo de sociedad?.

Las almazaras cooperativas andaluzas están superando estos años de crisis con una estrategia combinada: añadiendo a su principal fortaleza (su esencia organizativa) distintas medidas que mostramos a continuación.

REVESCO No 113 - MONOGRÁFICO: Crisis económica actual y sus posibles repercusiones en la economía social - ISSN: 1885-8031 - www.ucm.es/info/revesco 
a) La estructura de propiedad

Como indica el Informe del Consejo Superior de Cooperativas de Euskadi (2009), las cooperativas cuentan con instrumentos específicos (económico-legales) que no disponen otras formas jurídicas que les permiten hacer frente a estas situaciones complicadas de una forma distinta a la aplicada por las otras formas empresariales. Concretamente la Ley andaluza establece en su artículo 68 que de los resultados positivos se destinará, como mínimo, un 20\% al fondo de reserva obligatorio y de los resultados extracooperativos positivos, como mínimo, un $25 \%$. Este requerimiento legal unido a la práctica habitual de capitalización de retornos hace que los balances de las cooperativas estén más saneados que los de otras organizaciones. Así lo indican algunos de los sujetos entrevistados:

“...por eso la razón de que algunos particulares" hayan desaparecido es la forma de adelantar el cobro de la cosecha. Lo han hecho con anticipos de cosecha, con pólizas y después han tenido que vender por debajo del precio. Esto así no se sostiene y entonces muchos socios se han venido con nosotros. Por esa mala gestión”.

Algunos de los rasgos distintivos de la estructura de propiedad de la fórmula cooperativa como la primacía del socio frente al capital y/o la gestión democrática también hacen distinto el comportamiento de esta organización. La sociedad cooperativa almazarera es una empresa de empresarios. Y una vez deducidos los gastos, quien soporta la merma del beneficio es realmente el agricultor. Por tanto, en época de crisis el que finalmente ve reducidos sus ingresos es el propio socio, que además, en este tipo de entidades se caracteriza por ser una persona (en palabras de los entrevistados "prudente y ahorradora") aversa al riesgo. En los distintos discursos podemos apreciar estas ideas:

"La cooperativa es una empresa de empresarios, sus propietarios son los socios. Mientras que en una empresa privada si le va mal, quiebra la empresa porque tiene unos beneficios negativos y estos no pueden afrontar las pérdidas por mucho tiempo. Pero en la cooperativa son los socios los que obtienen las pérdidas directamente porque les liquidan a un precio menor, después de descontar los gastos de mantenimiento de la cooperativa. La

\footnotetext{
${ }^{11}$ Habitualmente en el sector oleícola existen dos tipos societarios principalmente: la cooperativa (que es la fórmula mayoritaria) y la S.L. A éste último tipo de entidad se conoce como "particulares" entre los miembros del sector.
}

REVESCO No 113 - MONOGRÁFICO: Crisis económica actual y sus posibles repercusiones en la economía social - ISSN: 1885-8031 - www.ucm.es/info/revesco 
cooperativa no quiebra. El dinero que hay lo reparten entre los socios, y si son pérdidas lo mismo".

Otra de las razones señaladas anteriormente ha sido la gestión democrática. El trato que de forma igualitaria se vive en el seno de una cooperativa genera confianza en la gestión. El pago a todos los socios del mismo precio por productos de igual calidad, o la transmisión de la misma información a todos los socios, serían ejemplos de justicia de procedimiento. Esta confianza disminuye el comportamiento oportunista y fomenta la cooperación, permite fijar un horizonte en la búsqueda del beneficio en las relaciones cooperativas a largo plazo y tiene implicaciones positivas en los resultados de la cooperativa en el mercado y en la eficiencia.

"El socio considera que la cooperativa es una garantía de cobro. Confía su patrimonio a la cooperativa, y no exigen el cobro inmediato, si no que espera a que la cooperativa pueda liquidar el aceite después de su venta”.

“...el beneficio redunda en todos, con lo cual se apuesta a un caballo seguro, y no cada año a un caballo ganador y eso es lo que hace que el socio continúe”.

b) El socio

El olivicultor tiene un arraigo especial a su actividad. El ser una tradición que va pasando de generación en generación y que en muchos casos no sea la principal actividad de la unidad familiar (lo que en cierto modo también permite que la economía doméstica se mantenga), son circunstancias que hacen que estos socios intenten soportar, como hemos indicado, etapas de recesión con bajadas de ingresos, a cambio de mantener su propiedad. En palabras de los entrevistados:

"La suerte que tienen las empresas agroalimentarias son los socios porque tienen una capacidad de aguante importante, eso les permite subsistir"

c) La relación socio-cooperativa

Como se ha señalado anteriormente la fortaleza de la relación que surge entre la empresa y el socio es una ventaja competitiva que perdura en el tiempo. Es una relación de confianza construida a lo largo del tiempo y basada en la gestión democrática. El socio puede

REVESCO No 113 - MONOGRÁFICO: Crisis económica actual y sus posibles repercusiones en la economía social - ISSN: 1885-8031 - www.ucm.es/info/revesco 
acceder a determinados recursos que conjuntamente generan capacidades que permiten a la cooperativa ganar o en cualquier caso mantener su competitividad.

De forma prioritaria los recursos que especialmente cubren las expectativas del socio son los relacionados con la extracción y cobro del aceite de oliva. El socio por sí solo no puede hacer frente a todas las actividades relacionadas con la extracción y comercialización del aceite. La cooperativa facilita la inversión que requiere todo el proceso, permitiendo a la vez que todos sus socios puedan hacer uso de las instalaciones. Cuando el socio se integra en una cooperativa puede disponer de unas instalaciones, equipos, maquinaria,...El acceso a este tipo de servicios le permite molturar la producción y obtener un precio de liquidación competitivo con equipos tecnológicamente avanzados.

La seguridad es otro de los intangibles especialmente valorados por el socio de una almazara cooperativa, entendiéndola como la percepción del socio de que las intenciones de la cooperativa a la que pertenece, le llevará a adoptar comportamientos que le permitirán conseguir los resultados previstos. Como anteriormente se indicó, estos resultados tienen que ver, fundamentalmente, con la garantía de venta y cobro de los productos aportados a la cooperativa, la obtención de precios adecuados de venta (compra) de los productos aportados (adquiridos) a la cooperativa y el acceso a diferentes servicios (formación, información, etc.) en buenas condiciones de calidad y precio.

"El socio continúa en la cooperativa por la garantía de que el dinero lo tiene seguro, porque es un mercado muy difícil el del aceite para especular o para negociar en él”

"Además el socio se garantiza que le van a hacer la liquidación real y no le van a dejar sin pagarle. Se garantiza el precio.”

También debemos reflejar la mayor relevancia que van adquiriendo los recursos relacionados con la información y el conocimiento. Los socios valoran muy positivamente que a través de la cooperativa puedan conocer nuevos tratamientos, nuevas aplicaciones en el cultivo, técnicas para mejorar sus plantaciones en aras de un producto de mejor calidad, etc. La información es un elemento importante para mejorar la competitividad del agricultor y la organización cooperativa suministra mucha información relevante.

Los directivos de estas empresas, así como los dirigentes de las organizaciones agrarias implicadas en el sector oleícola, se preocupan por la situación de los socios y REVESCO No 113 - MONOGRÁFICO: Crisis económica actual y sus posibles repercusiones en la economía social - ISSN: 1885-8031 - www.ucm.es/info/revesco 
consideran que los recursos clave a obtener a través de la cooperativa son la mejora de la calidad de su producto y la disminución de los costes. Todo ello se consigue con esta formación e información que se da a través de la cooperativa, así se implican en conseguir ideas innovadoras socialmente, que repercutan en las explotaciones agrícolas y en la mejora del producto.

El socio quizás en esta época de crisis controla más la gestión del Consejo, en palabras de un gerente "son más críticos" con las decisiones de los administradores, por la mala situación económica que atraviesan, sobre todo aquellos que en las épocas de crecimiento habían acometido importantes inversiones. No obstante, nos comentan que esa participación aunque algunas veces sólo se hace a través de críticas, puede traer ideas nuevas a la organización para mejorar la situación.

\subsection{Ampliación de servicios a los socios.}

Las organizaciones cooperativas almazareras que tienen mejores resultados es debido a que además de la labor de producción del aceite de oliva han ampliado sus servicios para ofrecer un valor añadido al socio. Estos servicios tienen una repercusión muy positiva en la valoración por parte del socio de su relación con la cooperativa, haciendo que dicha relación sea más sólida.

Este es el caso de los recursos de carácter financiero. Muchas cooperativas han creado secciones de crédito permitiendo al socio el acceso al crédito en mejores condiciones que a través del sistema financiero tradicional y gestionando un volumen de negocio realmente importante. Así se refleja en los discursos:

"Son importantes las secciones de crédito en las cooperativas almazareras. Tienen cuatrocientos millones de euros en sus secciones de crédito que representan el $60 \%$ del sector del valor de todas las cooperativas de crédito de Andalucía”.

También, como consecuencia de la actual crisis, se están modificando los procedimientos de liquidación de la cosecha al socio. En algunos casos, que el pago se hacía al final de año, ahora debido a que el agricultor puede tener dificultades para acceder al crédito a través de otras vías, se adelanta el pago en función de la evolución de las ventas en distintos momentos del año.

REVESCO No 113 - MONOGRÁFICO: Crisis económica actual y sus posibles repercusiones en la economía social - ISSN: 1885-8031 - www.ucm.es/info/revesco 
Otras de las medidas adoptadas ha sido la creación de centrales de compra (fitosanitarios, combustibles, aperos de labranza, herramientas...). Con la central de compras se facilita que el socio pueda adquirir los productos que necesita para su actividad a mejor precio que en el mercado. Así se recoge en uno de los discursos:

"Se vende gasoil, fitosanitarios, abonos y el margen de beneficio de todos los productos es para el socio. Tienen botas, herramientas, material para el campo. Se hacen compras al por mayor y eso representa un mayor beneficio para el socio”.

La creciente demanda del hueso de la aceituna como biocombustible también está haciendo que muchas almazaras incorporen este servicio de suministro tanto para sus socios como para personal externo a la cooperativa.

Otro servicio que también presta la cooperativa está relacionado con la asesoría de carácter laboral, jurídico, etc. En la mayoría de los casos son tareas relacionadas con las ayudas agrarias, producción integrada,... tratando de abaratar los costes de explotación de sus socios.

\subsection{Ajustes de plantilla}

La mayoría de las cooperativas almazareras que se han visto obligadas a tomar medidas extraordinarias para afrontar la crisis han adoptado acciones muy similares y en todos los casos con el único objetivo de mantener el empleo de los socios trabajadores, como se muestra a continuación:

“El año pasado llegaron a un acuerdo con el personal fijo discontinuo, no tenían trabajo para todos durante toda la campaña, la cooperativa propuso que los que tuviesen una mayor carga social (hijos, familia) los contrataban y a los demás no, haciendo un expediente temporal de regulación de empleo. Pero llegaron al acuerdo de repartirse el trabajo entre todos los trabajadores, ellos se repartieron el trabajo y trabajaron todos sin excepción”.

Esta es una diferencia considerable respecto a otro tipo de empresas, en las que el mantenimiento y la garantía del empleo de los trabajadores, no ha sido el principal objetivo de las medidas adoptadas.

REVESCO No 113 - MONOGRÁFICO: Crisis económica actual y sus posibles repercusiones en la economía social - ISSN: 1885-8031 - www.ucm.es/info/revesco 
Las almazaras cooperativas tienen una estructura laboral conformada por un número muy reducido de personal indefinido y un número variable de fijos discontinuos y realmente la capacidad de la plantilla es, generalmente, deficitaria en campaña.

\subsection{Crecimiento a través de la cooperación}

Otra de las características que diferencian a las cooperativas almazareras andaluzas es que muchas de ellas están asociadas a distintas federaciones, asociaciones o grupos de segundo grado. El hecho de que estén asociadas les permite tomar medidas de ayuda intercooperativa y les ayuda a no padecer de forma tan aislada las consecuencias de la crisis.

De esta forma, como se muestra en los discursos, la cooperación con otras cooperativas permite disponer de más recursos a través de los cuales pueden profesionalizar la gestión, utilizar una red interna de telefonía fija, recibir asesoramiento de todo tipo, servicio de recogida de residuos...

"Los que están en grupos cooperativos, quizás la liquidación no la hacen mejor que otras cooperativas, pero si gestionan otra serie de servicios que repercuten positivamente en el socio. Por ejemplo ahora en telefonía, los mil quinientos socios de Jaencoop y sus familias pueden hablar entre ellos sin coste alguno, al ser de la misma empresa”.

\subsection{Moderación en la inversión}

La mayoría de las almazaras cooperativas han disfrutado durante varios años de importantes ayudas, que desde la administración pública se facilitaban para la remodelación y adaptación del proceso productivo. Consecuencia de la crisis actual ha sido la reducción o desaparición de dichas ayudas y ahora las cooperativas tienen que hacer un mayor esfuerzo. $\mathrm{Su}$ respuesta es clara y prudente: "Innovación permanente, no pararse ante la crisis, pero ajustar las inversiones". Estas organizaciones tratan de reducir sus costes, adaptándose de la mejor forma posible a su entorno, buscando ser más competitiva y diferir las inversiones el máximo tiempo posible para que no sean demasiado gravosas para el socio.

"Esta cooperativa ha hecho hace un año una inversión de 30 millones de euros pero esta inversión se ha hecho teniendo en cuenta la situación del socio, intentando que la amortización de la inversión sea lo menos gravosa posible para el socio”.

REVESCO $\mathrm{N}^{\circ} 113$ - MONOGRÁFICO: Crisis económica actual y sus posibles repercusiones en la economía social - ISSN: 1885-8031 - www.ucm.es/info/revesco 
"La gestores de la cooperativa en momentos de crisis se deben poner los primeros, haciendo inversiones muy ajustadas. Ahora no se invierte en maquinaria más de lo necesario"

La cita anterior refleja la idea del ejemplo ético que deben dar los administradores en esta situación de crisis.

\section{CONCLUSIONES}

En este trabajo pretendemos ofrecer una visión cualitativa del efecto de la crisis sobre las cooperativas agroalimentarias, centrándonos en el caso de estudio de las cooperativas almazareras en Andalucía. Para ello nos hemos basado en la experiencia y conocimiento de los responsables de distintas cooperativas. Concretamente, hemos profundizado en aspectos como las medidas que éstas han adoptado para hacer frente a la crisis, que recursos reciben los agricultores de estas empresas para hacerles continuar en el negocio y como van generando capacidades que les permite mantenerse en una situación tan adversa como la actual.

A modo de conclusión, podemos afirmar que las sociedades cooperativas almazareras siguen, aún en épocas de crisis, constituyendo una herramienta fundamental en el sector y un recurso importante para el desarrollo de la actividad económica en las zonas rurales.

Actualmente estas empresas atraviesan un periodo complejo, propiciado no solo por la coyuntura económica actual, sino por la existencia de un mercado cada día más globalizado, concentrado y menos regulado. Los agricultores que las integran sufren una gran disminución de sus rentas, a pesar de su resistencia, su tendencia al ahorro y su flexibilidad ante las circunstancias adversas del entorno.

En este contexto, hemos advertido que los agricultores se ven apoyados en las sociedades cooperativas al ser empresas claramente orientadas a las personas y los valores del cooperativismo, con un modelo empresarial en el cual el socio y sus intereses en el proceso de producción y distribución se superponen al capital. A lo largo de su historia han sabido crear un capital social (Arcas et al., 2011; Rodríguez-Mondoño, 2012) que les ayuda a poder sobrevivir y seguir cumpliendo el objetivo que se marcaron desde su fundación ${ }^{12}$, que no es otro que dar ventaja en el mercado a los clientes, pequeños negocios, pequeños agricultores,

\footnotetext{
${ }^{12}$ Muchas de las sociedades cooperativas analizadas se crearon en la década de los cincuenta del siglo pasado REVESCO $\mathrm{N}^{\circ} 113$ - MONOGRÁFICO: Crisis económica actual y sus posibles repercusiones en la economía social - ISSN: 1885-8031 - www.ucm.es/info/revesco
} 
trabajadores, etc. Ya que se forman en respuesta al mercado para que éstos agentes mejoren su situación.

En este sentido, el principal apoyo de las cooperativas almazareras a sus socios ha sido conseguir dentro de sus posibilidades el mantenimiento del precio de liquidación a los socios de los productos entregados a la cooperativa, la garantía de cobro y de conservación de la relación calidad/precio en los pagos de los inputs. La volatilidad de los precios, ante la ausencia de mecanismos de intervención pública es algo habitual, lo que las hace cada vez más necesarias y a su vez genera el reto de la dimensión, no solo para conseguir una mayor concentración de la oferta ante la concentración de la demanda, sino por la necesidad de realizar inversiones que precisan economías de escala para poder ser viables.

Así hemos constatado que las empresas con mayor dimensión y que a su vez se han asociado con otras en cooperativas de segundo grado, han podido resistir mejor la crisis económica, porque les ha permitido aprovechar mejor las oportunidades de un mercado cada día más globalizado, al ser empresas que comparten objetivos y sus modelos de negocio son compatibles.

Igualmente, advertimos que estas organizaciones no solo pretenden resolver los problemas de mercado antes apuntados, sino que también pueden participar en el mercado de forma eficiente (Marcuello y Nachar, 2013), compitiendo bajo un esquema en el que se intenta colaborar internamente y, que bajo el apoyo de la confianza generado entre las personas que las componen, pueden asumir menores costes tanto económicos como sociales y generar externalidades positivas.

Lo anterior nos lleva a justificar la creación de un capital relacional en las sociedades cooperativas objeto de estudio, el cual podemos apreciar en diversos aspectos: en la flexibilidad para adaptarse a las circunstancias adversas; en el fomento de la intercooperación y el trabajo en red, como medio de supervivencia ante las nuevas circunstancias; y en la creación de empresas como entidades cualificantes de los socios que las componen.

Estas sociedades cooperativas las podemos ver como un claro ejemplo de innovación y emprendimiento social (Marcuello y Saz, 2008), debido a su comportamiento basado en los principios, tales como la naturaleza democrática, su estructura de gobierno, su cuidado por la

REVESCO No 113 - MONOGRÁFICO: Crisis económica actual y sus posibles repercusiones en la economía social - ISSN: 1885-8031 - www.ucm.es/info/revesco 
información y el aprendizaje, la importancia que le dan a las redes sociales y su enfoque hacia la comunidad.

No obstante, consideramos necesario apuntar líneas de actuación con vistas al futuro.

La crisis económica y financiera ha marcado un punto de inflexión, lo cual puede servir para rescatar valores como la ética empresarial, la transparencia en la gestión, la visión a largo plazo, el crecimiento sostenible y la cooperación como herramienta principal para la supervivencia. Todos estos valores son consustanciales al cooperativismo y se deben seguir haciendo valer por las sociedades cooperativas agroalimentarias en el futuro. Si nos fijamos en los países que cuentan con un sector agroalimentario más competitivo y dinámico son precisamente aquellos donde el cooperativismo alcanza una mayor relevancia (Juliá et al., 2010)

Futuras líneas de investigación debieran orientarse a contrastar de manera empírica los argumentos expuestos dentro del presente trabajo. Por ejemplo, incluyendo otros casos de estudio en otros sectores agroalimentarios, siendo esta una de las posibles limitaciones a considerar en nuestro trabajo, y haciéndolo en otros países. De lo anterior nace la oportunidad de entender mejor la sociedad cooperativa agroalimentaria y responder bajo qué condiciones y contextos significan un claro apoyo a la sociedad y a sus socios-proveedores.

\section{BIBLIOGRAFÍA}

ANDALEEB, S. S. (1996), An Experimental Investigation of Satisfaction and Commitment in Marketing Channels: The Role of Trust and Dependence. Journal of Retailing, 72 (1), p. 77-93.

ANDERSON, J. C. y NARUS, J. A. (1990), A model of distributor firm and manufacturer firm working partnership, Journal of Marketing, vol. 54, $\mathrm{n}^{\mathrm{o}}$ 1, p. $42-58$

ARCAS, N., HERNÁNDEZ, M. y MUNUERA, J. L. (2000), La relación entre las cooperativas agrarias de primer y segundo grado desde la perspectiva del marketing de relaciones y redes, CIRIEC-España, vol. 36, p. 179-202.

ARCAS, N. (2003), La relación entre las cooperativas agrarias de comercialización de primer y segundo grado: un modelo explicativo de sus características y resultados. Michigan USA: proquest company, p. 351 .

REVESCO No 113 - MONOGRÁFICO: Crisis económica actual y sus posibles repercusiones en la economía social - ISSN: 1885-8031 - www.ucm.es/info/revesco 
ARCAS, N. (Dir.). (2011), El gobierno de las cooperativas agroalimentarias. Factores de éxito. Fundación Cajamar. 101p. ISBN: 978-84-937759-7-1

AULAKH, P.S., KOTABE, M. y SAHAY, A. (1996), Trust and performance in cross-border marketing partnerships: a behavioral approach. Journal of International Business Studies, p. $1005-1032$.

BARTLETT, W., CABLE, J., ESTRIN, S., JENOS, D. y SMITH, S. (1992), Labor-Managed; Cooperatives and Private Firms in North Central Italy: An Empirical Comparison. Industrial and Labor Relations Review 46 (1), p. 103-118

BARNEY, J (1991), Firm Resources and Sustained Competitive. Advantage, Journal of Management, 17, 99-120.

BONTIS, N. (1996), There's a Price on your Head: Managing Intellectual Capital Strategically. Business Quarterly, Summer, p. 40-47.

BONTIS, N. (2001), Assessing knowledge assets: A review of the models used to measure intellectual capital. International Journal of Management Reviews, Vol. 3 No. 1, p. 41-60.

CALDERÓN, B. y CALDERÓN, M. J. (2012), ¿Cómo afrontan la crisis las cooperativas en España: comparativa de trayectorias laborales a partir de la Muestra Continua de Vidas Laborales”, CIRIEC-España, Revista de Economía Pública, Social y Cooperativa, $\mathrm{n}^{\circ}$ 76, p. 5-26.

CARDENAS, R Y VILAR, J. (2012), El sector internacional de elaboración de aceite de oliva. Jaén. Gea Westfalia Separator Ibérica.

CECOP-CICOPA Europa. (Junio 2011), A pesar de las dificultades, las cooperativas de industria y servicios, resisten a la crisis y sus consecuencias. Tercer informe anual sobre la crisis.

CIC-IADE (2003). Modelo Intellectus: Medición y gestión del capital intelectual. Madrid: Documento Intellectus $\mathrm{N}^{\circ} 5$.

CHETTY, S. (1996), The case study method for research in small and medium sized firms. International Small Business Journal, n ${ }^{\mathrm{o}}$ 15, pp.73-85.

CONSEJO SUPERIOR DE COOPERATIVAS DE EUSKADI (2009). Crisis económica y las cooperativas vascas. Disponible en internet: ekgk.coop/archivo_1/xihu1DVh_Microsoft\%20Word\%20\%20CRISIS\%20ECONOMICA\%20Y\%20LAS\%20COOPERATIVAS\%20VASCAS.pdf

REVESCO No 113 - MONOGRÁFICO: Crisis económica actual y sus posibles repercusiones en la economía social - ISSN: 1885-8031 - www.ucm.es/info/revesco 
CORNFORTH, C. y THOMAS, A. (1995), Les cooperatives de travail au Rouyaume Uni. Declin ou croissance?. Revue de Etudes Cooperatives, Mutualistee et Associatives, 255 (53).

EISENHARDT, K. M. (1989), Building theories form case study research. Academy of Management Review, vol. 4, nº 4, p. 532-550.

GARCÍA-GUTIÉRRÉZ, C. (2002), La empresa de participación: características que la definen. Virtualidad y sociedad de la información. CIRIEC-ESPAÑA. Revista de Economía Pública, Social y Cooperativa, no 40, p. 99-122. Disponible en Internet: www.ucm.es/info/revesco. ISSN. 1885-8031.

GUERRAS, L. A. (2004), Problemas organizativos en el proceso de la dirección estratégica. Universia Business Review, $\mathrm{n}^{\circ}$ 1, p. 116-125.

GUERRERI, G., NAZZARO, O. y ZEVI, A. (1992), La Economía Social en Italia”. En Monzón, J.L. y Defourny, J. (Dirs.), Economía Social, entre economía capitalista y economía pública, Valencia, CIRIEC-España y Ministerio de Trabajo y Seguridad Social, p. 157-198.

HERNÁNDEZ, M. y ARCAS, N. (2003), The effects of authoritative mechanism of coordination on market orientation in asymmetrical channel partnership, International Journal of research in marketing, vol. 20 (2), p. 133-152.

HERNÁNDEZ, M. J., MARQUEZ, A. M., y PEDROSA, C. (2012), “Cooperación e internacionalización en el sector del aceite de oliva como estrategia para la competitividad: el caso Hojiblanca", Cuadernos de Estudios Agroalimentarios, Vol. 4, p.103-117

IGLESIAS, V., DEL RIO, A. B. y TRESPALACIOS, J. A. (1998), Factores determinantes y consecuencias de la confianza entre las empresas en sus relaciones en los canales de distribución. VIII Congreso nacional de Acede Empresa y economía institucional, p.1-14.

JONES, D. y KALMI, P. (2009), Trust, Inequality and the Size of the Co-operative Sector: Cross-Country Evidence. Annals of Public and Cooperative Economics, 80 (2), pp. 165195.

JUliA, J. F; GARCIA, G.; MELIA, E. y GALlEGO, L. P. (2010), Los factores de competitividad de las cooperativas líderes en el sector agroalimentario europeo. Fundación Cajamar.

LEJARRIAGA, G. (2002), Las empresas de participación como base del contenido de la "economía social” en el marco de la Unión Europea. En Vargas, A. \& Lejarriaga, G.

REVESCO No 113 - MONOGRÁFICO: Crisis económica actual y sus posibles repercusiones en la economía social - ISSN: 1885-8031 - www.ucm.es/info/revesco 
(coord.), Las empresas de participación en Europa: el reto del siglo XXI, Escuela de Estudios Cooperativos, p. 83-116.

Ley 14/2011, de 23 de diciembre, de Sociedades Cooperativas Andaluzas

MARCUELLO, C. y SAZ, M. I. (2008), Los principios cooperativos facilitadores de la innovación. Un modelo teórico. REVESCO. Revista de Estudios Cooperativos, $\mathrm{n}^{\circ}$ 94, $1^{\circ}$ cuatrimestre, p. 59-79. Disponible en Internet: www.ucm.es/info/revesco. ISSN. 18858031.

MARCUELLO, C. y NACHAR, P. (2013), La sociedad cooperativa: motivación y coordinación. Un análisis desde las teorías económicas de la empresa y la economía social. REVESCO. Revista de Estudios Cooperativos, n 110, p. 192-222. Disponible en Internet: www.ucm.es/info/revesco. ISSN. 1885-8031.

NAHAPIET, J. y GHOSHAL, S. (1998), Social capital, intelectual capital and the organizational advantage, Academy of Management Review, vol 23, n², p. 242-266

NOVKOVIC, S. (2008), Defining the co-operative difference. The Journal of SocioEconomics, n' 37 , p. 2168-2177.

RODRÍGUEZ, P. (2004), El capital social en el nuevo institucionalismo económico, en Actas del $2^{\circ}$ Evento Internacional Desarrollo Económico e Instituciones. Grupo Eumed, Universidad de Málaga.

RODRÍGUEZ-MONDROÑO, P. (2012), Análisis relacional del capital social y el desarrollo de los sistemas productivos regionales. REDES-Revista hispana para el análisis de redes sociales, vol. 23, nº 9, p. 261-290.

RYAN, B.; SCAPENS, W. y THEOBALD, M. (1992), Research method and methodology in finance and accounting. London: Academic Press, Harcourt Brace Jovanovich Publishers.

SPEAR, R. (2000), The Co-operative Advantage, Annals of Public and Co-operative Economics, Carfax, Oxford.

SPENDER, J. C. y GRANT, R. M. (1996), Knowledge and the firm: overview. Strategic Management Journal, vol. 17, p. 5-9.

STRATEGOR (1995). Estrategia, estructura, decisión, identidad. Masson, Barcelona.

STEWART, T. A. (1997), Intellectual Capital: The New Wealth of Organizations. Doubleday Currency, New York.

TEECE, David J. (1987), Profiting from Technological Innovation: Implications for Integration, Collaboration, Licensing, and Public Policy'. In The competitive challenge, Teece, David J. (Ed.), Cambridge MA: Ballinger Pub. Co., pp. 185-219.

REVESCO No 113 - MONOGRÁFICO: Crisis económica actual y sus posibles repercusiones en la economía social - ISSN: 1885-8031 - www.ucm.es/info/revesco 
VILLARREAL, O. y LANDETA, J. El estudio de casos como metodología de investigación científica en economía de la empresa y dirección estratégica. XXI Congreso Anual AEDEM, Universidad Rey Juan Carlos, Madrid, 6-8 Junio.

WILLIAMSON, O. E. (1975), Markets and Hierarchies: Analysis and Antitrust Implications. New York: Free Press.

YIN, R. K. (1989), Case study research, design and methods. Sage Publications, Newbury Park.

YOUNDT M. A., SUBRAMANIAM M. y SNELL S. A. (2004), Intellectual capital profiles: an examination of investments and returns. Journal of Management Studies, $\mathrm{n}^{\circ}$ 41, p. 335361.

REVESCO No 113 - MONOGRÁFICO: Crisis económica actual y sus posibles repercusiones en la economía social - ISSN: 1885-8031 - www.ucm.es/info/revesco 